\title{
On the logic and framework of enterprise management reform in the era of artificial intelligence
}

\author{
Ciwen Pang ${ }^{1, *}$ \\ ${ }^{1}$ Shanghai University of international business and Economics, 201620 Shanghai, China
}

\begin{abstract}
With the development of science and technology, artificial intelligence technology is gradually improving. At present, artificial intelligence has become an important trend in the development of science and technology. The emergence of the era of artificial intelligence brings great opportunities and challenges to enterprises. Under the background of artificial intelligence, the personnel composition and working mode of enterprises will also change greatly, which means that the management mode of enterprises also needs to be changed accordingly. Based on this, starting from the development trend of artificial intelligence, this paper analyzes the logical framework of enterprise management reform in the era of artificial intelligence, hoping to provide some reference for enterprise management, so as to promote the development of Chinese enterprises in the era of artificial intelligence.
\end{abstract}

\section{INTRODUCTION}

From the emergence of computer to the artificial intelligence, it only experienced several decades. In recent years, artificial intelligence related news often appears in people's eyes. For example, "alphago" defeated the world champion in go, and "Xiaodu" robot performed well in the "strongest brain" variety show [1]. With the emergence of these news, we gradually realize that the era of artificial intelligence is really coming. It is no longer just the imagination in science fiction movies, but is about to become a reality. Especially for enterprises, they begin to realize that artificial intelligence will bring great changes to the market. At the same time, the emergence of artificial intelligence is a stimulus to the management of enterprises. How to deal with this kind of stimulation is a problem that enterprise managers need to think about.

\section{Development history of artificial intelligence}

The emergence of artificial intelligence is not accidental, when science and technology develop to a certain extent, people will start to study deeper technology, will start to study the direction of life creation, artificial intelligence is in this context. At present, it is generally believed that the beginning of artificial intelligence was the Dartmouth conference in 1956.[2] At the beginning, people's research on artificial intelligence is still in a superficial stage. Since the 1980 s, the development of artificial intelligence in the world has entered a new era, people began to have a more in-depth study and understanding of artificial intelligence. At present, artificial intelligence has gradually entered the public field of vision.

For the specific definition of artificial intelligence, in fact, there is not a very complete definition. With the continuous development of artificial intelligence and scientists' in-depth understanding of artificial intelligence, the definition of artificial intelligence is constantly changing. At the beginning, the definition of artificial intelligence is a science that enables machines to do what people can do [3]. Later people think that artificial intelligence refers to the development of thinking machines that can imitate human intelligence. Until now, people generally believe that artificial intelligence is a machine that can carry out all kinds of human activities and has certain learning ability and human intelligence. Although the specific definition of artificial intelligence must not be defined, but from the perspective of the development of artificial intelligence, artificial intelligence has two main characteristics, the first is intelligence, it needs to be able to replace human beings in some social activities. Secondly, we need to have certain thinking and learning ability, and be able to learn and grow by ourselves. It is precisely because of these characteristics of artificial intelligence that most countries are very cautious in the face of artificial intelligence.

From the development history of artificial intelligence, we can infer the general development trend of artificial intelligence. The current AI is just at the stage of looking like. In other words, these robots can carry out some simple activities and conversations, but they have no ability to learn. The development of artificial intelligence will go through two stages: strong artificial intelligence and super artificial intelligence. Strong artificial intelligence means that the artificial

* Corresponding author: $1446155278 @$ qq.com 
intelligence robot has a certain learning ability, can constantly learn in some aspects, and achieve the goal of working instead of human beings. Super artificial intelligence means that artificial intelligence has been completely similar to human beings, and can have faster calculation and discrimination ability. Of course, this is a very ideal state, and because artificial intelligence, especially super artificial intelligence, involves the creation of "life", in our country, or in most countries, it is very cautious. Before we have a definite grasp, most countries still focus on weak artificial intelligence.

\section{The necessity of introducing artificial intelligence into enterprise management}

Financial management mode refers to the financial management mode that exists in the overall management framework of the enterprise and is designed for the overall financial objectives of the enterprise. The system needs to classify and analyze the complex information of the enterprise. Compared with artificial intelligence, this is a common function of $\mathrm{F}$, which is indispensable for financial management of enterprises. As far as pattern recognition is concerned, fuzzy pattern recognition and statistical pattern recognition are two important methods of pattern recognition. Fuzzy pattern recognition corresponds to the financial management, which is suitable for large-scale information and data processing, and can greatly improve the efficiency of financial management. However, because the selection standard is only one approximate value, and the accuracy is 7 poor, this kind of fuzzy financial management mode still needs to be improved. Statistical pattern is to describe the studied pattern with feature vector, and divide the features with various methods in order to achieve the goal of pattern. In the financial management, this can improve the efficiency of awareness, but also reduce the error rate, compared with fuzzy financial management, statistical financial management mode is more common.

This paper analyzes the concept of enterprise management, which refers to the organization, coordination, planning and other functions of enterprises in order to carry out production and operation activities. In recent years, with the rapid development of information technology, a series of highly technical software systems such as CRM and ERP have been formed, which play a synergistic role in data collection. With the increasing application of network technology, the above-mentioned systems are gradually converging to promote the formation of a broader and deeper system. The application of artificial intelligence in enterprise management has gone beyond the scope of computing and consulting services. Its future development direction is to subvert the traditional information exchange mode and promote the penetration of artificial intelligence in various fields of the company.

From the perspective of corporate structure, the purpose of optimizing and formulating the organizational structure is to maximize the company's own profits. As for the organizational structure, the process of its intelligent development is the process of continuously strengthening the goals set by the organization. It is not just to reduce the humanistic intervention, but to reduce the number of jobs with the characteristics of repetitive labor, automatically analyze the needs of consumers and work, so as to complete the work plan and optimize the application of resources. Compared with human beings with emotion and error risk, big data analysis by robots and support from relevant platforms can reduce the error rate and improve the service value rapidly. In response to emergencies, it can automatically search for solutions, which provides a strong guarantee for normal operation, and is shown as a 24-hour uninterrupted service state. As a manager, he can have more time to participate in the production process and change the previous pyramid like management structure to a flat one. The focus of enterprise management has also shifted from monitoring the details of structural movement to focusing on the overall data.

In the past, the controllable and planned operation mode provided favorable conditions for the formation of high-quality and small-scale professional team. However, most of the members were less sensitive to the changes in the external market and only paid attention to the changes around them. The individual development space was obviously limited, resulting in lower competitiveness, Lack of independent innovation ability. Although the application of artificial intelligence technology has no substitute conditions for the middle and senior management, it can have an impact on many fields such as capital management, technology and circulation, and indirectly play a guiding role in the future development of enterprises. Artificial intelligence can analyze the past data, gain experience, transform to strategy, evaluate and judge the relationship between data comprehensively and objectively, and then guide management. For example, the sales link of an enterprise can have a direct impact on the efficiency and operation of the whole company, which is related to the future development of the company. Using the artificial intelligence management platform, it can automatically remind the sales risk, formulate the sales plan, and remind the technical deficiencies, so as to provide guidance for the development of new products and give managers a broader vision, While paying attention to the core business, ensure the efficient operation of the enterprise.

\section{The influence of artificial intelligence on enterprise management}

The emergence of artificial intelligence, especially the emergence of intelligent robots, has a great impact on enterprises. At present, some manufacturing enterprises have realized semi automation, which means that they use a lot of intelligent robots. In this case, their management style is bound to change. The influence of artificial intelligence on enterprise management is mainly reflected in two aspects.

The first is the influence of artificial intelligence technology on the development of enterprises. Compared 
with computer technology, artificial intelligence technology is more advanced. Moreover, because of its intelligence, it can analyze and solve problems by itself in some stages. For enterprises, this kind of intelligence will greatly reduce management costs and improve management efficiency. For example, the current emergence of "smart logistics" is the impact of artificial intelligence on enterprises. At the same time, in the process of enterprise development, the flow of personnel is inevitable. The recruitment and turnover rate of enterprises has always been a very big problem. Through artificial intelligence technology, we can analyze the internal e-mail and various behaviors of employees, so as to judge the possibility of employee turnover and help enterprises better prepare. At present, artificial intelligence technology can also help enterprises to quickly screen and match online resumes, reducing the time of enterprise recruitment.

Secondly, the impact of AI development on enterprises. Artificial intelligence has an excellent development space, therefore, now most enterprises are involved in the artificial intelligence industry. Artificial intelligence can indeed bring greater convenience to enterprise management, and also can reduce the operating costs of enterprises to a certain extent. However, when enterprises create and use artificial intelligence, they must pay attention to social ethics. We should bear in mind that the emergence of artificial intelligence only serves human beings, not replaces them.

\section{Analysis of logic and framework of enterprise management change in artificial intelligence Era}

The framework of enterprise management change in the era of artificial intelligence is as follows:

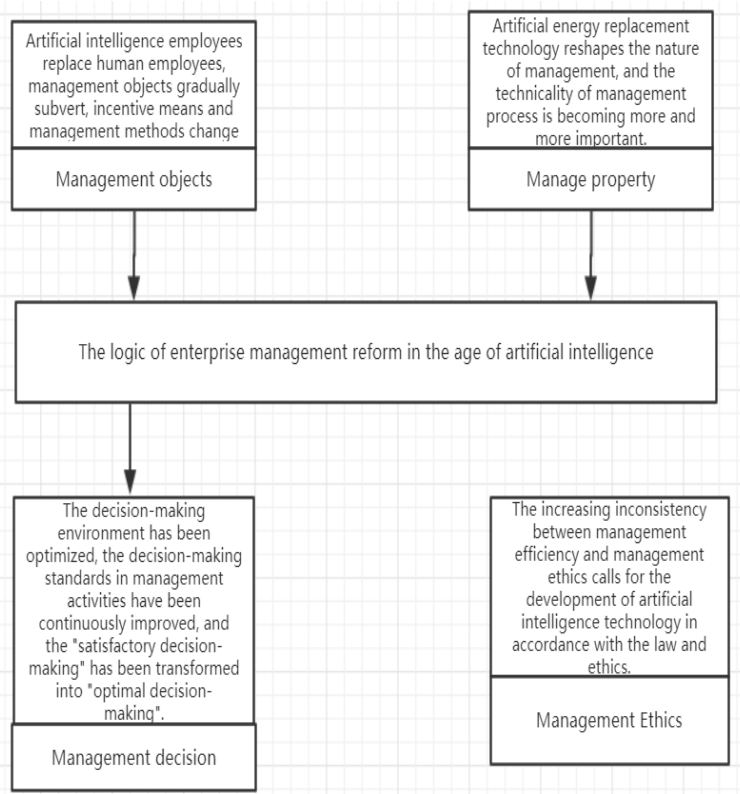

Fig. 1. The framework of enterprise management reform in artificial intelligence Era

\subsection{Changes in the composition of the managed}

In the traditional enterprise management, both the manager and the managed are human beings. Therefore, in the enterprise management, people have been making and implementing management rules with people as the center. Indeed, in the traditional business model, all the work is done by people. In this case, it is very correct to manage people. But in the era of artificial intelligence, besides people, there are also artificial intelligence who work for enterprises. It can provide more support for enterprises in many ways. Especially in some repetitive jobs, the advantages of human beings are gradually replaced by artificial intelligence robots. In this context, the managers of enterprises have changed. In the process of management, enterprises not only need to manage people, but also need to do a good job in the management of artificial intelligence robots. In the process of managing the miracle man of artificial intelligence, the enterprise must realize that it is impossible to manage artificial intelligence with the set of manager. Although artificial intelligence can imitate human beings, it is not a real human being. It is difficult to sense any emotion. In this case, the management mode of enterprises should be divided into two types: one is the management of human beings, the other is the management of artificial intelligence. Secondly, artificial intelligence does not need to sleep like human beings, it only needs regular maintenance. Of course, with the continuous development of artificial intelligence, perhaps one day, artificial intelligence can have the ability of management. In order to better deal with this situation, enterprise managers need to constantly improve themselves, do a good job in the management of artificial intelligence and human employees, and prevent themselves from being replaced by artificial intelligence.

\subsection{Changes in management attributes}

Management attribute is to analyze the characteristics of management from the perspective of philosophy. Generally speaking, management needs both theory and practice. At present, the generally accepted attribute of management is that management is the unity of science and art [4]. Scientificity refers to the theoretical support of management, which requires the management theory to be scientific and reasonable. And artistry refers to the practice of management process, need enough flexibility and flexibility, in order to better face the problems in management. This kind of management attribute is originally very good, it is the unity of theory and practice. But in the era of artificial intelligence, this attribute is insufficient. In the era of artificial intelligence, managers not only need to manage human beings, but also need to manage artificial intelligence. Artificial intelligence, unlike human beings, can automatically repair and adjust. This means that in the management of artificial intelligence staff, not only need the scientific management, but also need to be technical. This technicality is based on artificial intelligence. When there are problems in artificial intelligence, managers need to be able to solve them. It can be said that in the 
era of artificial intelligence, the technology of managers will become more and more important. This technology is not management technology, but intelligent technology.

\subsection{Changes in decision making}

Decision is a very important part of management. If the decision of managers is wrong, it will lead to the whole management work. In the traditional management decision-making, most of them make decisions based on the managers' own understanding of various information and the experience of managers. This decision can be limited by the managers' understanding of information and their own experience. However in the era of artificial intelligence, the decision-making of managers will be rational, which means that management decision-making will be more satisfying. This situation is mainly the huge information processing system of artificial intelligence. Managers can understand various information of enterprises through artificial intelligence, and then decide better decision by artificial intelligence calculation. This decision-making method is not limited to decision makers, which makes the optimal decision information possible. In addition, with the continuous development of artificial intelligence, it will be more and more familiar with human thinking. In this case, managers can also use artificial intelligence to simulate the thinking process of human beings, so as to put forward-looking decisions. There are two main advantages to use AI to help decision-making. First, the comprehensiveness of information. AI can show all kinds of information of the enterprise in a very comprehensive way. Secondly, controllability. Compared with human, all kinds of behavior modes of AI are in a controllable state. In this controllable state, decision-making will become more convenient.

\subsection{The challenge of management ethics}

There are three main indicators for the management of enterprises, namely, efficiency, effect and benefit. Efficiency refers to work efficiency, and effect is the goal of behavior. Benefit is the standard of test results. Any enterprise will use these three indicators in the process of management. However, in the era of artificial intelligence, these three indicators are facing ethical challenges. Under the background of artificial intelligence, management means are also gradually intelligent, but some enterprises use this intelligent means to carry out the management mode which is not in line with humanity. For example, in the news before, "the Environmental Health Department of a city monitors the working status of sanitation workers through distribution of smart bracelets or smart phones", this way does improve the efficiency of the group, but it is not humanized. How to balance intelligence, humanity and regularization is the problem that enterprises need to solve. Secondly, it is the challenge of law and morality. The emergence of artificial intelligence, such as unmanned vehicles, has brought great challenges to the formulation of traffic regulations. At the same time, the branch of artificial intelligence: genetic science must conform to social ethics. The emergence of artificial intelligence is to serve the society and the enterprise, not to bring the invisible harm to the enterprise and society.

\section{Conclusion}

The emergence of artificial intelligence will make great changes in the management framework of enterprises. For enterprises, they need to change the current management mode in the era of artificial intelligence to better cope with the changes of the times. The reform of enterprise management mode can be carried out from the aspects of management object, management attribute, decision-making mode and management ethics. In short, the use of artificial intelligence, can better achieve enterprise management, but at the same time, artificial intelligence also brings greater challenges to enterprise management. Enterprises need to balance the opportunities and challenges.

\section{References}

1. Deng Yalin, Jiang Jisheng. The change of enterprise personnel management in the era of artificial intelligence $[\mathrm{J}]$. Enterprise reform and management, 2018, (12): 82,84.

2. Lu Jingjing. Research on enterprise management change in the era of artificial intelligence [j]. Caixun, 2018, (10): 97.

3. Ruan Manzhi, Wang Jiachen. Research on the development and transformation of Chinese private enterprises in the era of artificial intelligence [J]. Economic Research Guide, 2019, (12): 22-23.

4. Deng Yalin, Jiang Jisheng. The change of enterprise personnel management in the era of artificial intelligence $[\mathrm{J}]$. Enterprise reform and management, 2018, (12): 82,84.

5. Ge Yaxiu, Chen Kaiyu. The change of enterprise human resource management under artificial intelligence [J]. Economic Research Guide, 2020, (3): 124-126150. 